\section{A cost effective solution for beautiful restorations}

The MyCrown fully integrated CAD/CAM system uses guided software with five simple steps that streamline the production of various restorative solutions - including crowns, veneers, inlays, onlays and small bridges.

Featuring a touchscreen monitor and an advanced intraoral scanner, MyCrown eliminates the need for traditional impression materials, as practitioners are able to capture detailed digital scans of a patient's oral cavity. These 2D and 3D images are available for immediate review and editing, which facilitates the delivery of a supreme-fitting prosthesis.

The two-spindle system of MyCrown's sophisticated milling unit then fabricates the proposed restoration with speed and accuracy. Many practitioners are astounded with the system's high performance, which can produce a single crown in an average of just 12 minutes - without ever sacrificing on quality.

Moreover, MyCrown is optimised to mill from restoration blocks according to your preference, so you are not limited to a specific manufacturer or material.

For more information, visit www.my-crown.co.uk or call 03339873007.

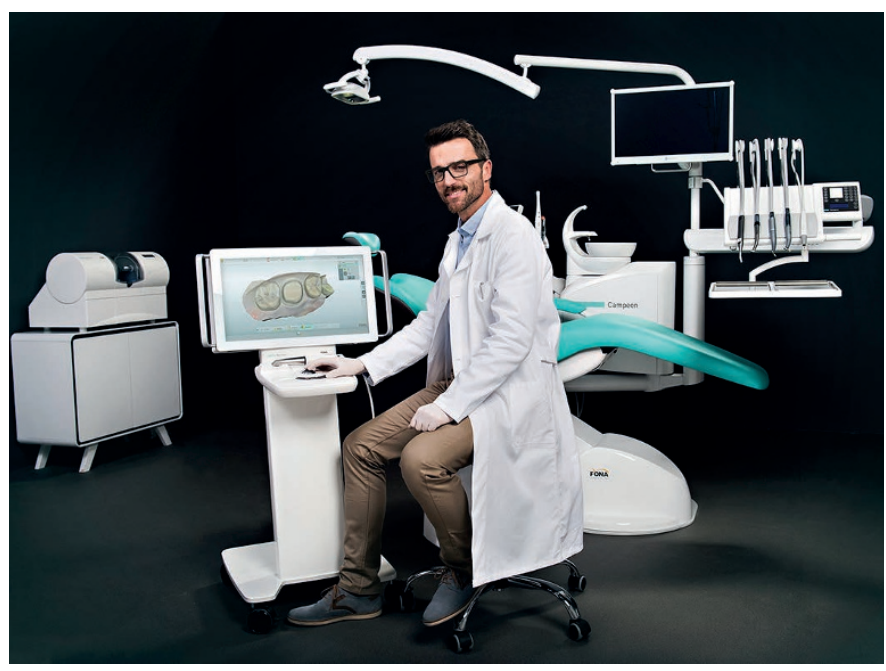

\section{Information at your fingertips}

The field of dental implantology is constantly evolving. Make sure you keep up to date with all of the latest developments by becoming a member of the Association of Dental Implantology (ADI).

Members of the ADI receive hard copies quarterly of the EDI Journal - a resource that is specifically aimed at professionals who focus on dental implantology and those who want to learn more. ADI members also receive access to the highly rated online Clinical Oral Implants Research journal (COIR). Membership also grants you unlimited access to OvidSP, a collection of past articles from trusted scientific resources.

For those looking for even more information, membership to the ADI grants you access to the ADI Members-only Facebook group, where you can talk to and discuss topics with other members, keeping you in the loop and giving you the chance to make new contacts within the field.

For more information please visit www.adi.org.uk.

\section{Financial cover against illness}

When it comes to protecting against illness and time off work, there are two types of insurance available: critical illness cover and income protection. Critical illness pays out a single tax-free lump sum against a number of serious illnesses and medical conditions, while income protection provides a monthly payment in the event of sickness, injury or accident.

For optimal protection it is always advisable to contact a specialist Independent Financial Adviser (IFA) who can work closely with you to establish your needs and help you select an appropriate policy.

At money4dentists the expert team of IFAs have over half a century of experience in helping dentists with critical illness cover and income protection. Together with an unrivalled knowledge of the various products available from a range of insurers, you are sure to find one or more policies that suit your needs.

For more information please call 08453455060 or 0754DENTIST, email info@money4dentists.com or visit www.money4dentists.com.

\section{Help patients to express themselves}

Lifestyle plays a huge part in oral health decisions, and thanks to Curaprox health-conscious patients can now experience oral freshness and whitening like never before, in a way that appeals to their unique natures and decision making.

The recently launched Be You range of whitening toothpastes in six exclusive, vibrant flavours and colours is the latest addition to Curaprox's line of innovative oral health products.

These new toothpastes come in an array of flavours and colours such as tangy Grapefruit with Bergamot, Watermelon and a sure-to-be-favourite Gin and Tonic with Persimmon. Whatever the personality of your patient, they're sure to find one that suits and offers the chance to express themselves.
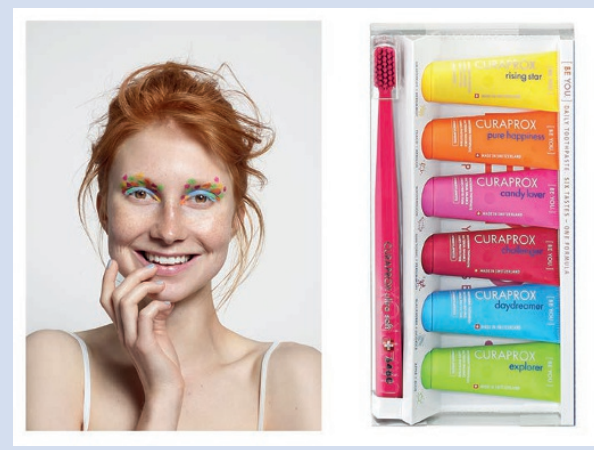

All products within the range include $950 \mathrm{ppm}$ fast-acting fluoride, hydroxylapatite to remineralise the teeth, glucose oxidase to support the natural enzymatic process and whiten teeth, and xylitol to prevent the growth of cavity-causing bacteria. They also feature Echinacea, devil's claw and pennywort to help soothe and regenerate mucus membranes and gingiva as well as reduce inflammation.

For more information please call 01480 862084, email info@ curaprox.co.uk or visit www.curaprox.co.uk. 